\title{
ASYMPTOTIC STABILITY OF THE CAUCHY AND JENSEN FUNCTIONAL EQUATIONS
}

\author{
ANNA BAHYRYCZ, ZSOLT PÁLES, AND MAGDALENA PISZCZEK
}

\begin{abstract}
The aim of this note is to investigate the asymptotic stability behaviour of the Cauchy and Jensen functional equations. Our main results show that if these equations hold for large arguments with small error, then they are also valid everywhere with a new error term which is a constant multiple of the original error term. As consequences, we also obtain results of hyperstability character for these two functional equations.
\end{abstract}

\section{Introduction}

The stability theory of the classical Cauchy and Jensen functional equations has attracted a lot of attention in the last decades. Historically, this theory was stimulated by a question of S. M. Ulam [31, though the first result of this kind is due to Gy. Pólya and G. Szegó [25]. The majority of the results concentrates on proving that if a function satisfies a given functional equation approximately, then it approximates a function which is the exact solution of the given functional equation. To demonstrate this phenomenon, we recall D. H. Hyers' classical theorem ([16]) which states that if, $X$ is a linear space and $Y$ is a Banach space and, for some $\varepsilon \geq 0$, a function $f: X \rightarrow Y$ is $\varepsilon$-additive, i.e.,

$$
\|f(x)+f(y)-f(x+y)\| \leq \varepsilon \quad(x, y \in X),
$$

then there exists an additive function $g: X \rightarrow Y$ such that $f$ is $\varepsilon$-close to $g$, i.e.,

$$
\|f(x)-g(x)\| \leq \varepsilon \quad(x \in X) .
$$

Motivated by this result several contributions have been obtained since then ([1, 8, 26, 21]). For the interested reader, we recommend the following books and surveys: Ger [15], Forti [13], Czerwik [10], Hyers, Isac and Rassias [17]. There are at least four significantly different approaches to obtain stability theorems. The first one, the so-called direct method, or iterative method was already invented by Hyers in [16] (see also [6], [14, [24, [28]). Results using the technique of invariant means (over amenable semigroups) were first proved by L. Székelyhidi [29] (see also [2], 3], 44). The third method is to use variants of the Hahn-Banach separation theorem or sandwich theorems, or more generally selections theorems, see [23], [22]. The most recently discovered method is to use fixed point theorems suggested by L. Cădariu and V. Radu [11, [12] (see also [9]).

In the present note, we do not apply any of the general patterns to obtain stability theorems. Instead, we assume that the stability of the functional equation holds only for large values of the variables and we deduce that the stability holds on the entire domain. Such results explicitely or implicitely have already been obtained by S.-M. Jung [18], by L. Losonczi [20], and by F. Skof [27]. To formulate the main results of this paper, the most convenient structures for the domain and the codomain of the given functions are metric abelian groups. A triple $(X,+, d)$ is

\footnotetext{
Date: August 6, 2018.
}

Key words and phrases. functional equation; stability; asymptotic stability; metric group.

This research of the second author was supported by the Hungarian Scientific Research Fund (OTKA) Grant K111651. 
called a metric abelian group if $(X,+)$ is an abelian group which is equipped with a translation invariant metric $d$. In this case we define $\|x\|_{d}:=d(x, 0)$ and we call $\|\cdot\|_{d}$ the norm induced by the metric $d$. Then $\|\cdot\|_{d}$ is an even subadditive function on $X$. The standard example for a metric abelian group is the additive group of a normed space. We note that, in general, the norm on a metric abelian group is not necessarily positively homogeneous. By the subadditivity of the norm, the inequality $\|2 x\|_{d} \leq 2\|x\|_{d}$ is always valid, however the equality may fail.

\section{Results on the Stability}

Theorem 1. Let $(X,+, d)$ and $(Y,+, \rho)$ be metric abelian groups such that $X$ is unbounded by $d$. Let $\varepsilon \geq 0$ and assume that $f: X \rightarrow Y$ possesses the following asymptotic stability property

$$
\limsup _{\min \left(\|x\|_{d},\|y\|_{d}\right) \rightarrow \infty}\|f(x+y)-f(x)-f(y)\|_{\rho} \leq \varepsilon,
$$

then

$$
\|f(x+y)-f(x)-f(y)\|_{\rho} \leq 5 \varepsilon \quad \text { for all } x, y \in X .
$$

Furthermore, provided that (11) holds, the constant $5 \varepsilon$ is the smallest possible in (2).

Proof. Let $\eta>\varepsilon$ be arbitrary. Then, by the asymptotic stability property (11) of $f$, there exists $r>0$ such that, for all $x, y \in X$ with $\|x\|_{d} \geq r,\|y\|_{d} \geq r$,

$$
\|f(x+y)-f(x)-f(y)\|_{\rho}<\eta .
$$

Let $x, y \in X$ be fixed. Using the unboundedness of $X$, choose $u \in X$ first and then $v \in X$ such that

$$
\|u\|_{d} \geq r+\|x\|_{d} \quad \text { and } \quad\|v\|_{d} \geq r+\|x\|_{d}+\|y\|_{d}+\|u\|_{d} .
$$

Then, one can easily see that

$$
\min \left(\|u\|_{d},\|v\|_{d},\|u+v\|_{d},\|x-u\|_{d},\|y-v\|_{d},\|x+y-u-v\|_{d}\right) \geq r .
$$

Applying (3) five times, we get

$$
\begin{array}{r}
\|f(x-u)+f(u)-f(x)\|_{\rho}<\eta, \\
\|f(y-v)+f(v)-f(y)\|_{\rho}<\eta, \\
\|f(x+y-u-v)-f(x-u)-f(y-v)\|_{\rho}<\eta, \\
\|f(u+v)-f(u)-f(v)\|_{\rho}<\eta, \\
\|f(x+y)-f(x+y-u-v)-f(u+v)\|_{\rho}<\eta .
\end{array}
$$

By, the triangle inequality, we obtain

$$
\begin{aligned}
\|f(x+y)-f(x)-f(y)\|_{\rho} \leq & \|f(x-u)+f(u)-f(x)\|_{\rho} \\
& +\|f(y-v)+f(v)-f(y)\|_{\rho} \\
& +\|f(x+y-u-v)-f(x-u)-f(y-v)\|_{\rho} \\
& +\|f(u+v)-f(u)-f(v)\|_{\rho} \\
& +\|f(x+y)-f(x+y-u-v)-f(u+v)\|_{\rho} \\
< & 5 \eta .
\end{aligned}
$$

Taking the limit $\eta \rightarrow \varepsilon$ in the last inequality, we arrive at (2), which was to be proved. 
To show that $5 \varepsilon$ is the best constant in (2), let $x_{0} \in X \backslash\{0\}$ be an arbitrary element and define a function $f: X \rightarrow \mathbb{R}$ as follows:

$$
f(x)= \begin{cases}\varepsilon, & \text { for } x \in X \backslash\left\{x_{0}\right\}, \\ 3 \varepsilon, & \text { for } x=x_{0} .\end{cases}
$$

Then, for all $x, y \in X$ with $\|x\|_{d},\|y\|_{d}>\left\|x_{0}\right\|_{d}$, we have $f(x)=f(y)=\varepsilon$ and $f(x+y) \in\{\varepsilon, 3 \varepsilon\}$. Hence, for large values of $x$ and $y$,

$$
|f(x+y)-f(x)-f(y)|=\varepsilon,
$$

which implies the validity of (11). On the other hand, using that $2 x_{0} \neq x_{0}$, we obtain

$$
\sup _{x, y \in X}|f(x+y)-f(x)-f(y)| \geq\left|f\left(2 x_{0}\right)-2 f\left(x_{0}\right)\right|=5 \varepsilon
$$

proving that the constant on the right hand side of (2) cannot be smaller than $5 \varepsilon$.

Our next result is related to the asymptotic stability of the Jensen functional equation.

Theorem 2. Let $(X,+, d)$ and $(Y,+, \rho)$ be metric abelian groups such that $X$ is uniquely 2divisible and unbounded by $d$. Let $\varepsilon \geq 0$ and assume that $f: X \rightarrow Y$ possesses the following asymptotic stability property

$$
\limsup _{\min \left(\|x\|_{d},\|y\|_{d}\right) \rightarrow \infty}\left\|2 f\left(\frac{x+y}{2}\right)-f(x)-f(y)\right\|_{\rho} \leq \varepsilon
$$

then

$$
\left\|4 f\left(\frac{x+y}{2}\right)-2 f(x)-2 f(y)\right\|_{\rho} \leq 4 \varepsilon \quad \text { for all } x, y \in X .
$$

Furthermore, provided that (44) holds, the constant $4 \varepsilon$ is the smallest possible in (5).

Proof. Let $\eta>\varepsilon$ be arbitrary. Then, applying (44), there exists $r>0$ such that, for all $x, y \in X$ with $\|x\|_{d} \geq r,\|y\|_{d} \geq r$,

$$
\left\|2 f\left(\frac{x+y}{2}\right)-f(x)-f(y)\right\|_{\rho}<\eta .
$$

Let us fix $x, y \in X$ and choose $u \in X$ such that

$$
\|u\|_{d} \geq r+\|x\|_{d}+\|y\|_{d} .
$$

Then, as $\min \left(\|x+u\|_{d},\|y+u\|_{d},\|x-u\|_{d},\|y-u\|_{d}\right) \geq r$, we get the following four inequalities

$$
\begin{array}{r}
\left\|2 f\left(\frac{x+y}{2}\right)-f(x+u)-f(y-u)\right\|_{\rho}<\eta, \\
\left\|2 f\left(\frac{x+y}{2}\right)-f(x-u)-f(y+u)\right\|_{\rho}<\eta, \\
\|f(x+u)+f(x-u)-2 f(x)\|_{\rho}<\eta, \\
\|f(y+u)+f(y-u)-2 f(y)\|_{\rho}<\eta .
\end{array}
$$

Thus, by the triangle inequality, we obtain

$$
\begin{aligned}
&\left\|4 f\left(\frac{x+y}{2}\right)-2 f(x)-2 f(y)\right\|_{\rho} \\
& \leq\left\|2 f\left(\frac{x+y}{2}\right)-f(x+u)-f(y-u)\right\|_{\rho}+\left\|2 f\left(\frac{x+y}{2}\right)-f(x-u)-f(y+u)\right\|_{\rho} \\
& \quad+\|f(x+u)+f(x-u)-2 f(x)\|_{\rho}+\|f(y+u)+f(y-u)-2 f(y)\|_{\rho} \\
&<4 \eta .
\end{aligned}
$$


Upon taking the limit $\eta \rightarrow \varepsilon$, the desired inequality (5) results.

To verify that $4 \varepsilon$ is the best constant in (5), it suffices to consider the function $f: X \rightarrow \mathbb{R}$ defined by

$$
f(x)= \begin{cases}0, & \text { for } x \in X \backslash\left\{x_{0},-x_{0}, 0\right\}, \\ -\frac{\varepsilon}{2}, & \text { for } x \in\left\{x_{0},-x_{0}\right\}, \\ +\frac{\varepsilon}{2}, & \text { for } x=0\end{cases}
$$

where $x_{0} \in X \backslash\{0\}$ is an arbitrary element. Then, for all $\|x\|_{d},\|y\|_{d}>\left\|x_{0}\right\|_{d}$,

$$
\left|2 f\left(\frac{x+y}{2}\right)-f(x)-f(y)\right| \in\{0, \varepsilon\},
$$

wich shows that (4) is satisfied. On the other hand,

$$
\sup _{x, y \in X}\left|4 f\left(\frac{x+y}{2}\right)-2 f(x)-2 f(y)\right| \geq\left|4 f(0)-2 f\left(x_{0}\right)-2 f\left(-x_{0}\right)\right|=4 \varepsilon,
$$

proving that the constant on the right hand side of (5) cannot be smaller than $4 \varepsilon$.

Taking $\varepsilon=0$ in Theorems 1 and 2, we can directly obtain the following corollaries.

Corollary 3. Let $(X,+, d)$ and $(Y,+, \rho)$ be metric abelian groups such that $X$ is unbounded by d. If $f: X \rightarrow Y$ satisfies

$$
\limsup _{\min \left(\|x\|_{d},\|y\|_{d}\right) \rightarrow \infty}\|f(x+y)-f(x)-f(y)\|_{\rho}=0,
$$

then

$$
f(x+y)=f(x)+f(y), \quad x, y \in X .
$$

Corollary 4. Let $(X,+, d)$ and $(Y,+, \rho)$ be metric abelian groups such that $X$ is uniquely 2divisible and unbounded by $d$. If $f: X \rightarrow Y$ satisfies

$$
\limsup _{\min \left(\|x\|_{d},\|y\|_{d}\right) \rightarrow \infty}\left\|2 f\left(\frac{x+y}{2}\right)-f(x)-f(y)\right\|_{\rho}=0,
$$

then

$$
4 f\left(\frac{x+y}{2}\right)=2 f(x)+2 f(y), \quad x, y \in X
$$

\section{RESUlts ON THE HYPERSTABILITY}

Theorem 5. Let $(X,+, d)$ and $(Y,+, \rho)$ be metric abelian groups such that $2 X$ is unbounded by d. Let $\varphi: \mathbb{R}_{+}:=[0, \infty) \rightarrow \mathbb{R}$ such that $\lim _{t \rightarrow \infty} \varphi(t)=\infty$ and let $f: X \rightarrow Y$ satisfy

$$
\limsup _{\min \left(\|x\|_{d},\|y\|_{d}\right) \rightarrow \infty} \varphi\left(\|x-y\|_{d}\right) \cdot\|f(x+y)-f(x)-f(y)\|_{\rho}<\infty,
$$

then

$$
f(x+y)=f(x)+f(y) \quad \text { for all } x, y \in X .
$$

Proof. According to (6), there exist constants $r>0$ and $K>0$ such that, for $x, y \in X$ with $\|x\|_{d} \geq r$ and $\|y\|_{d} \geq r$

$$
\varphi\left(\|x-y\|_{d}\right) \cdot\|f(x+y)-f(x)-f(y)\|_{\rho}<K .
$$

Let $\varepsilon>0$ be arbitrary and choose $R>0$ such that, for all $t \geq R$,

$$
\varphi(t) \geq \frac{5 K}{\varepsilon} .
$$


Then, for $x, y \in X$ with $\|x\|_{d} \geq r,\|y\|_{d} \geq r$ and $\|x-y\|_{d} \geq R$, we have

$$
\|f(x+y)-f(x)-f(y)\|_{\rho}<\frac{\varepsilon}{5} .
$$

Let $x, y \in X$ be fixed. In view of the unboundedness of $2 X$, we can choose $u \in X$ such that

$$
\|2 u\|_{d} \geq 2 r+R+2\|x\|_{d}
$$

and then we can choose $v \in X$ satysfying

$$
\|2 v\|_{d} \geq 2 r+2 R+2\|x\|_{d}+2\|y\|_{d}+2\|u\|_{d}
$$

Then, using the trivial inequality $\|2 z\|_{d} \leq 2\|z\|_{d}$ (which is the consequence of the subadditivity of $\|\cdot\|_{d}$ ), we easily get

$$
\begin{array}{r}
\min \left(\|u\|_{d},\|v\|_{d},\|u+v\|_{d},\|x-u\|_{d},\|y-v\|_{d},\|x+y-u-v\|_{d}\right) \geq r, \\
\min \left(\|x-2 u\|_{d},\|y-2 v\|_{d},\|x-y-u+v\|_{d},\|u-v\|_{d},\|x+y-2(u+v)\|_{d}\right) \geq R .
\end{array}
$$

Thus, using inequality (7) on the domain indicated, we obtain the following five inequalities

$$
\begin{array}{r}
\|f(x-u)+f(u)-f(x)\|_{\rho}<\frac{\varepsilon}{5}, \\
\|f(y-v)+f(v)-f(y)\|_{\rho}<\frac{\varepsilon}{5}, \\
\|f(x+y-u-v)-f(x-u)-f(y-v)\|_{\rho}<\frac{\varepsilon}{5}, \\
\|f(u+v)-f(u)-f(v)\|_{\rho}<\frac{\varepsilon}{5}, \\
\|f(x+y)-f(x+y-u-v)-f(u+v)\|_{\rho}<\frac{\varepsilon}{5} .
\end{array}
$$

Adding up these inequalities side by side and using the triangle inequality (as in the proof of Theorem 1), we get

$$
\|f(x+y)-f(x)-f(y)\|_{d} \leq \varepsilon .
$$

Since $\varepsilon>0$ was arbitrary, this inequality implies that $f$ is additive.

We cannot omit the assumption that $2 X$ is unbounded by $d$ in Theorem 5 , Indeed, if $\sup _{x \in X}\|2 x\|_{d}=K<\infty$, then the function $f: X \rightarrow X$ defined by

$$
f(x)= \begin{cases}x, & x \in X \backslash\{0\} \\ a, & x=0\end{cases}
$$

where $a \neq 0$, for $x, y \in X \backslash\{0\}$ satisfies

$$
\|x-y\|_{d} \cdot\|f(x+y)-f(x)-f(y)\|_{d}= \begin{cases}0 & \text { if } x+y \neq 0 \\ \|2 x\|_{d} \cdot\|a\|_{d} & \text { if } x+y=0\end{cases}
$$

Hence

$$
\limsup _{\min \left(\|x\|_{d},\|y\|_{d}\right) \rightarrow \infty}\|x-y\|_{d} \cdot\|f(x+y)-f(x)-f(y)\|_{d} \leq K \cdot\|a\|_{d}<\infty,
$$

which means that (6) is fulfilled with $\varphi(t):=t$. On the other hand, $f$ is not additive.

Theorem 6. Let $(X,+, d)$ and $(Y,+, \rho)$ be metric abelian groups such that $X$ is uniquely 2divisible and $2 X$ is unbounded by $d$. Let $\varphi: \mathbb{R}_{+} \rightarrow \mathbb{R}$ such that $\lim _{t \rightarrow \infty} \varphi(t)=\infty$ and let $f: X \rightarrow Y$ satisfy

$$
\limsup _{\min \left(\|x\|_{d},\|y\|_{d}\right) \rightarrow \infty} \varphi\left(\|x-y\|_{d}\right) \cdot\left\|2 f\left(\frac{x+y}{2}\right)-f(x)-f(y)\right\|_{\rho}<\infty,
$$


then

$$
4 f\left(\frac{x+y}{2}\right)=2 f(x)+2 f(y) \quad \text { for all } x, y \in X .
$$

Proof. Similarly as in the proof of Theorem [5, it follows from (8) that there exist constants $r>0$ and $K>0$ such that, for $x, y \in X$ with $\|x\|_{d} \geq r$ and $\|y\|_{d} \geq r$,

$$
\varphi\left(\|x-y\|_{d}\right) \cdot\left\|2 f\left(\frac{x+y}{2}\right)-f(x)-f(y)\right\|_{\rho}<K .
$$

Let $\varepsilon>0$ be arbitrary and choose $R>0$ such that, for all $t \geq R$,

$$
\varphi(t) \geq \frac{4 K}{\varepsilon} .
$$

Then, for $x, y \in X$ with $\|x\|_{d} \geq r,\|y\|_{d} \geq r$ and $\|x-y\|_{d} \geq R$, we have

$$
\left\|2 f\left(\frac{x+y}{2}\right)-f(x)-f(y)\right\|_{\rho}<\frac{\varepsilon}{4} \text {. }
$$

Let $x, y \in X$ be fixed and, using the unboundedness of $2 X$, choose $u \in X$ such that

$$
\|2 u\|_{d} \geq 2 r+R+2\|x\|_{d}+2\|y\|_{d} .
$$

Then, by the triangle inequality, it follows that

$$
\begin{array}{r}
\min \left(\|x+u\|_{d},\|y+u\|_{d},\|x-u\|_{d},\|y-u\|_{d}\right) \geq r \\
\min \left(\|2 u\|_{d},\|x-y+2 u\|_{d},\|x-y-2 u\|_{d} \geq R .\right.
\end{array}
$$

Applying these conditions for the appropriate choice of variables, (10) implies the following four inequalities:

$$
\begin{array}{r}
\left\|2 f\left(\frac{x+y}{2}\right)-f(x+u)-f(y-u)\right\|_{\rho}<\frac{\varepsilon}{4}, \\
\left\|2 f\left(\frac{x+y}{2}\right)-f(x-u)-f(y+u)\right\|_{\rho}<\frac{\varepsilon}{4}, \\
\|f(x+u)+f(x-u)-2 f(x)\|_{\rho}<\frac{\varepsilon}{4}, \\
\|f(y+u)+f(y-u)-2 f(y)\|_{\rho}<\frac{\varepsilon}{4} .
\end{array}
$$

Thus

$$
\left\|4 f\left(\frac{x+y}{2}\right)-2 f(x)-2 f(y)\right\|_{\rho}<\varepsilon
$$

and as $\varepsilon>0$ was arbitrary, (9) holds.

We complete this paper by providing an example of an unbounded metric abelian group $X$ such that $2 X$ is bounded.

Example 7. Let

$$
X:=\left\{\left(a_{1}, a_{2}, \ldots\right): a_{i} \in\{0,1\}, i \in \mathbb{N}, \exists_{n_{0} \in \mathbb{N}} \forall_{i \geq n_{0}} a_{i}=0\right\} .
$$

For $\left(a_{1}, a_{2}, \ldots\right),\left(b_{1}, b_{2}, \ldots\right) \in X$, we define

$$
\left(a_{1}, a_{2}, \ldots\right)+\left(b_{1}, b_{2}, \ldots\right)=\left(\left(a_{1}+{ }_{2} b_{1}, a_{2}+{ }_{2} b_{2}, \ldots\right),\right.
$$

(where the operation $+_{2}$ is the additon modulo 2 on $\{0,1\}$ ) and

$$
d\left(\left(a_{1}, a_{2}, \ldots\right),\left(b_{1}, b_{2}, \ldots\right)\right)=\sum_{i=1}^{\infty} \frac{a_{i}+{ }_{2} b_{i}}{i} .
$$


Then $(X,+, d)$ is an unbounded metric abelian group but $2 X=\{(0,0, \ldots)\}$, hence $2 X$ is trivially bounded.

\section{REFERENCES}

[1] T. Aoki. On the stability of the linear transformation in Banach spaces. J. Math. Soc. Japan, 2:64-66, 1950.

[2] R. Badora. On some generalized invariant means and their application to the stability of the Hyers-Ulam type. Ann. Polon. Math., 58(2):147-159, 1993.

[3] R. Badora, R. Ger, and Zs. Páles. Additive selections and the stability of the Cauchy functional equation. ANZIAM J., 44(3):323-337, 2003.

[4] R. Badora, Zs. Páles, and L. Székelyhidi. Monomial selections of set-valued maps. Aequationes Math., 58(3):214-222, 1999.

[5] A. Bahyrycz and M. Piszczek. Hyperstability of the Jensen functional equation. Acta Math. Hungar., 142(2):353-365, 2014.

[6] K. Baron and P. Volkmann. On functions close to homomorphisms between square symmetric structures. Seminar LV (http://www.mathematik.uni-karlsruhe.de/ semlv/), (14):12 pp. (electronic), 2002.

[7] Z. Boros, Zs. Páles, and P. Volkmann. On stability for the Jensen equation on intervals. Aequationes Math., 60:291-297, 2000.

[8] D. G. Bourgin. Classes of transformations and bordering transformations. Bull. Amer. Math. Soc., 57:223-237, 1951.

[9] J. Brzdęk, J. Chudziak, and Zs. Páles. A fixed point approach to stability of functional equations. Nonlinear Anal., 74(17):6728-6732, 2011.

[10] S. Czerwik. Functional equations and inequalities in several variables. World Scientific Publishing Co., Inc., River Edge, NJ, 2002.

[11] L. Cădariu and V. Radu. The stability of Jensen's functional equation: a fixed point approach. Automat. Comput. Appl. Math., 11(1):27-32 (2003), 2002.

[12] L. Cădariu and V. Radu. Fixed points and the stability of Jensen's functional equation. JIPAM. J. Inequal. Pure Appl. Math., 4(1), 2003.

[13] G.-L. Forti. Hyers-Ulam stability of functional equations in several variables. Aequationes Math., 50(12):143-190, 1995.

[14] G.-L. Forti. Comments on the core of the direct method for proving Hyers-Ulam stability of functional equations. J. Math. Anal. Appl., 295(1):127-133, 2004.

[15] R. Ger. A survey of recent results on stability of functional equations. In Proc. of the 4th International Conference on Functional Equations and Inequalities, page 5-36, Cracow, 1994. Pedagogical University of Cracow.

[16] D. H. Hyers. On the stability of the linear functional equation. Proc. Natl. Acad. Sci. U.S.A., 27:222-224, 1941.

[17] D. H. Hyers, G. Isac, and Th. M. Rassias. Stability of functional equations in several variables. Progress in Nonlinear Differential Equations and their Applications, 34. Birkhäuser Boston Inc., Boston, MA, 1998.

[18] S.-M. Jung. Hyers-Ulam-Rassias stability of Jensen's equation and its application. Proc. Amer. Math. Soc., 126(11):3137-3143, 1998.

[19] Z. Kominek. On a local stability of the Jensen functional equation. Demonstratio Math., 22(2):499-507, 1989.

[20] L. Losonczi. On the stability of Hosszú's functional equation. Results Math., 29(3-4):305-310, 1996.

[21] L. Maligranda. A result of Tosio Aoki about a generalization of Hyers-Ulam stability of additive functions-a question of priority. Aequationes Math., 75(3):289-296, 2008.

[22] K. Nikodem, Zs. Páles, and Sz. Wąsowicz. Abstract separation theorems of Rodé type and their applications. Ann. Polon. Math., 72(3):207-217, 1999.

[23] Zs. Páles. Generalized stability of the Cauchy functional equation. Aequationes Math., 56(3):222-232, 1998.

[24] Zs. Páles, P. Volkmann, and R. D. Luce. Hyers-Ulam stability of functional equations with a square-symmetric operation. Proc. Natl. Acad. Sci. USA, 95(22):12772-12775 (electronic), 1998.

[25] Gy. Pólya and G. Szegô. Aufgaben und Lehrsätze aus der Analysis, Vol. I. Springer-Verlag, Berlin, 1925.

[26] Th. M. Rassias. On the stability of the linear mapping in Banach spaces. Proc. Amer. Math. Soc., 72(2):297-300, 1978.

[27] F. Skof. On the approximation of locally $\delta$-additive mappings. Atti Accad. Sci. Torino Cl. Sci. Fis. Mat. Natur., 117(4-6):377-389 (1986), 1983.

[28] Á. Száz. An instructive treatment and some natural extensions of a set-valued function of Páles. Math. Pannon., 24(1):77-108, 2013. 
[29] L. Székelyhidi. Remarks on Hyers's theorem. Publ. Math. Debrecen, 34(1-2):131-135, 1987.

[30] Ja. Tabor and Jó. Tabor. Stability of the Cauchy equation on an interval. Aequationes Math., 55(1-2):153-176, 1998.

[31] S. M. Ulam. A Collection of Mathematical Problems. Interscience Tracts in Pure and Applied Mathematics, no. 8. Interscience Publishers, New York-London, 1960.

E-mail address, A. Bahyrycz: bah@up.krakow.pl

Department of Mathematics, Pedagogical University of Cracow, Cracow, Poland

E-mail address, Zs. Páles: pales@science.unideb.hu

Institute of Mathematics, University of Debrecen, Hungary

E-mail address, M. Piszczek: magdap@up.krakow.pl

Department of Mathematics, Pedagogical University of Cracow, Cracow, Poland 